\title{
Observational window functions in planet transit searches
}

\author{
Kaspar von Braun ${ }^{1}$ and David R. Ciardi ${ }^{1}$ \\ ${ }^{1}$ Michelson Science Center \\ California Institute of Technology \\ 770 South Wilson Ave. \\ Pasadena, CA 91125, USA \\ email: kaspar@ipac.caltech.edu; ciardi@ipac.caltech.edu
}

\begin{abstract}
Window functions describe, as a function of orbital period, the probability that an existing planetary transit is detectable in one's data for a given observing strategy. We show the dependence of this probability upon several strategy and astrophysical parameters, such as length of observing run, observing cadence, length of night, and transit duration. The ability to detect a transit is directly related to the intrinsic noise of the observations. In our simulations of the window function, we explicitly address non-correlated (white) noise and correlated (red) noise and discuss how these two different noise components affect window functions in different manners.
\end{abstract}

Keywords. methods: statistical, planetary systems, time

\section{Introduction}

The signal-to-noise ratio (SNR) of a planetary transit detection in a given photometric light curve (here defined as simply time versus magnitude, irrespective of presence of absence of variability) can, in the simplest case, be approximated by:

$$
S N R=\frac{\operatorname{depth}}{\sigma} \sqrt{n}
$$

where depth is the transit depth in magnitudes, $\sigma$ represents the photometric measurement uncertainty in magnitudes per data point (assumed here to be the same for all data points), and $n$ equals the number of data points observed during transit. The fundamental assumption in this equation is the absence of any statistically correlated (red) noise, i.e., the only source of noise is random (white) noise.

White noise is defined as noise that is uncorrelated from data point to data point; typical sources are photon noise and sky background noise. White noise decreases with increasing brightness of the observed target. Red noise is defined as noise that is correlated from data point to data point; it is not necessarily removed through standard differential or ensemble photometry techniques. Typical sources of red noise may be weather, seeing changes, or tracking errors. It does not change as a function of target magnitude.

As Pont (2006) and Pont et al. (2006) initially pointed out, the assumption of the presence of only white noise in equation 1.1 is incorrect, and one needs to account for the presence of red noise in the calculation of the SNR and the corresponding expected yields for transit surveys, given survey and astrophysical parameters. A more detailed description of the transit detection SNR which includes both uncorrelated (white) and correlated (red) noise components is given by Pont et al. (2006): 


$$
S N R=\frac{\text { depth }}{\sqrt{\frac{1}{n^{2}} \sum_{i, j} C_{i j}}}=\frac{\text { depth }}{\sqrt{\frac{\sigma^{2}}{n}+\frac{1}{n^{2}} \sum_{i \neq j} C_{i j}}},
$$

where $C_{i j}$ is the covariance matrix. In equation 1.2 , the elements $C_{i j}$ represent the correlation coefficents between the $i$-th and $j$-th measurement obtained during transit. All diagonal elements $C_{i i}=\sigma_{i}^{2}$ are not correlated with other measurements and thus represent the uncorrelated or white noise uncertainties in the $i$-th measurement. It is furthermore assumed that $\sigma_{i}=\sigma$ for all values of $i$.

In order to make the above equation more practically calculable, Pont et al. (2006) assume that statistical correlation among data points from different transits will be much weaker than among data points observed during the same transit. They furthermore separate the total noise into a purely uncorrelated (white) component $\sigma_{w}$ and a purely correlated (red) component $\sigma_{r}$ and use these to derive an approximation of equation 1.2:

$$
S N R=\sqrt{\frac{(\operatorname{depth} \cdot n)^{2}}{\sum_{k=1}^{N_{t r}}\left[n_{k}^{2}\left(\frac{\sigma_{w}^{2}}{n_{k}}+\sigma_{r}^{2}\right)\right]}},
$$

where $n$ is the total number of data points observed during all transits, $N_{t r}$ is the total number of transits observed, $n_{k}$ is the number of data points observed during the $k$-th transit, and $\sigma_{w}$ and $\sigma_{r}$ are the white and red noise components, respectively.

By means of equation 1.3, the SNR can be regarded as a function of transit survey strategy and astrophysical parameters (see §2). If it exceeds a certain threshold value, then an existing transiting planet is detectable in the data. The window function indicates the probability, as a function of orbital period, that the SNR ratio exceeds this threshold and thus produces a detectable planet transit.

We briefly outline our algorithm and methods in $\S 2$, show respective influences of varying white and red noise components in $\S 3$, and examine the effects of various survey strategy and astrophysical parameters in $\S 4$. We summarize and conclude in $\S 5$.

\section{Algorithm and Input Parameters}

The algorithm used for the calculation of the window functions uses input on observing cadence as well as the number and typical length of night to generate an observing time line. From the user-provided stellar and planetary radii, it calculates transit depth and duration according to the equations in Seager \& Mallén-Ornelas (2003), thereby assuming a central transit. For each orbital period, a family of light curves is generated for a range of starting phase angles. Finally, the magnitude of the white and red noise components ( $\sigma_{w}$ and $\sigma_{r}$, as defined in $\S 1$ and Pont et al. 2006) are specified in the input.

In the simulations, the number of data points per transit $\left(n_{k}\right)$, number of transits $\left(N_{t r}\right)$ and total number of data points within all transits $(n)$ are tracked. For every light curve, the SNR (equation 1.3) is calculated. If, for a given phase angle, the SNR exceeds $\mathrm{SNR}_{\text {threshold }}$, a transit is considered "detected". The probability of detection $\left(\mathrm{P}_{\text {Detection }}\right)$ for a given orbital period is simply the ratio of phase angles for which a transit was detected to the total number of phase angles.

We attempted to choose the input values such that they resemble the ones found in transit surveys described in the literature, though our choice is probably slightly tilted toward surveys that do not have the luxury of having dedicated survey facilities. Thus, typical observational parameter values are: tens of minutes for the observing cadence, tens of nights for observing run length, and few to ten hours for the typical time of 

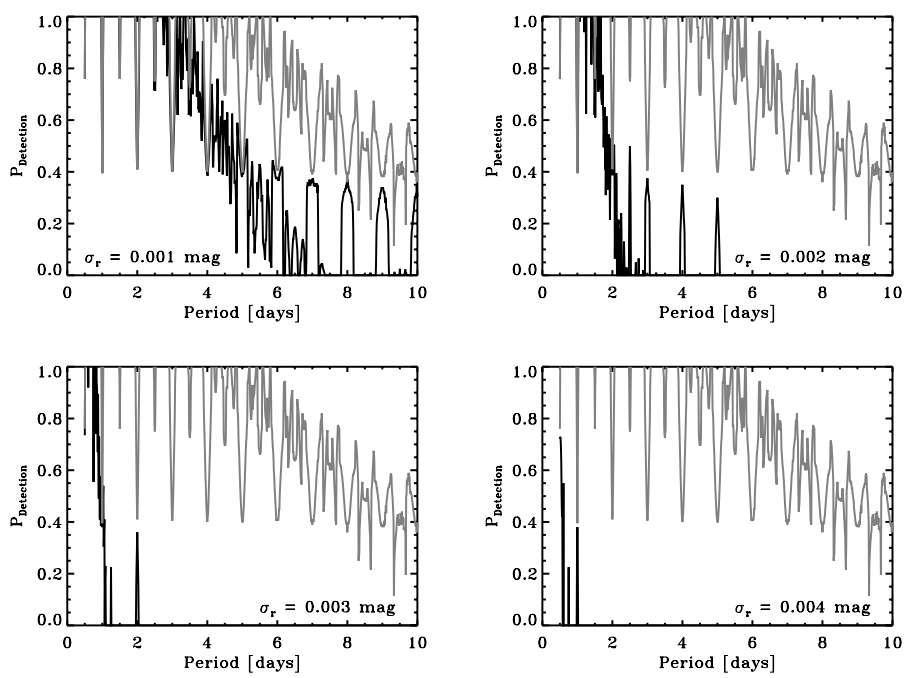

Figure 1. $\sigma_{w}$ constant $(5 \mathrm{mmag}), \sigma_{r}$ variable. The grey line (identical in all four panels) corresponds to the detection probability in the absence of red noise $\left(\sigma_{r}=0\right)$. The black line shows the same probability for varying levels of $\sigma_{r}$, indicated in each of the four panels. Other input parameters are $\mathrm{SNR}_{\text {threshold }}=10$, stellar/planetary radius $=1.0 / 0.1 \mathrm{R}_{\text {sun }}, 30$ consecutive observing nights, 8 hours of observing per night, and an observing cadence of 10 minutes.

observation spent during one night on the monitored target. Astrophysical parameter values are assumed to be around 1.0 and 0.1 solar radii for the parent star and orbiting planet, respectively, resulting in a transit depth of 0.01 mag (transit duration depends on period, but typical duty cycles are in the $1 \%$ to few $\%$ range), and a few millimagnitudes (mmag) for $\sigma_{w}$ and $\sigma_{r}$. The threshold SNR is set to 10 .

We note that, in contrast to other window function calculations, we only use the SNR criterion to quantify detections, and do not require that, e.g., a full transit be contained in the data or that data from at least two or more transits be sampled.

\section{Red Noise versus White Noise}

Due to their low signal depths $(<1 \%)$, transits can typically only be detected for the bright stars in one's sample. For the brightest stars within a survey, photon noise generally dominates the white noise. However, Pont et al. (2006) show that red noise (independent of target brightness) is particularly important in this regime. A more quantitative justification of this statement can be found in Aigrain \& Pont (2007), based on arguments initially presented in Pepper \& Gaudi (2005). In Figs. 1 and 2, we show the effects of varying amounts of white and red noise on the window function. Input parameters (as described in $\S 2$ ) are given in the captions of all figures. The grey line in the figure panels always indicates the window function in the absence of any correlated noise $\left(\sigma_{r}=0\right)$. In comparison, the black line indicates the window function resulting from presence of both white and red noise $\left(\sigma_{r} \neq 0\right)$.

Fig. 1 shows the window function for a constant white noise term $\sigma_{w}=5$ mmag in combination with a variable red noise term. Pont et al. (2006) quote the OGLE survey's brightest stars' $\sigma_{w}$ to be around $5 \mathrm{mmag}$ and $\sigma_{r}$ to be around $3-4 \mathrm{mmag}$. Kane et al. (2007) quote the WASP survey's $\sigma_{r}$ to be $2-3 \mathrm{mmag}$, which reduces to $1.5 \mathrm{mmag}$ after detrending using the SYSREM algorithm described in Tamuz et al. (2005). It is 

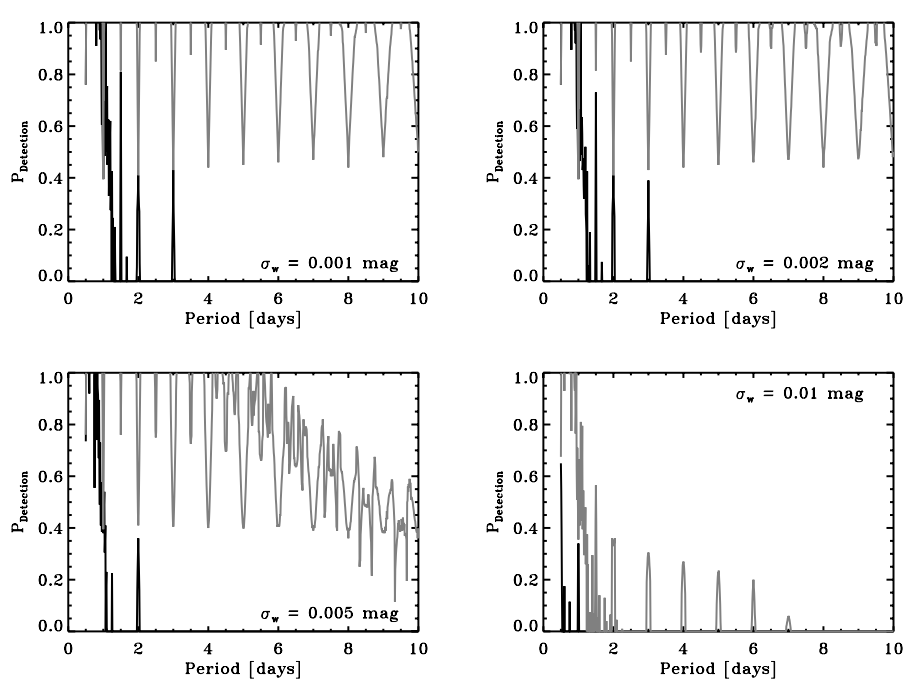

Figure 2. $\sigma_{r}$ constant $(3 \mathrm{mmag}), \sigma_{w}$ variable (shown in each of the four panels). As in Fig. 1 , the grey line corresponds to the detection probability in the absence of red noise, for the purpose of comparison with the actual window function when red noise is present (black line). Other input parameters are the same as in Fig 1. Clearly, the $\sigma_{r}$ dominates, even in the case of exceptionally low white noise.

impressive to see the decrease in the detection efficiency even for small changes in $\sigma_{r}$ in Fig. 1, illustrating the importance of the use of detrending algorithms for transit survey data, such as SYSREM (Tamuz et al. 2005) or TFA (Kovács et al. 2005).

Fig. 2 shows the window function for a constant red noise term $\sigma_{r}=3 \mathrm{mmag}$ in combination with a variable $\sigma_{w}$. For comparison purposes, the case for $\sigma_{r}=0$ is also shown (grey line) in each panel.

These Figures show that even small amounts of red noise will be the dominant factor in any calculation concerning transit detectability or survey yields, at least before any detrending is applied.

\section{The Influence of Observing Strategy and Astrophysical Parameters on the Window Function}

The careful consideration and simulation of individual aspect of transit survey strategy can significantly improve the detection efficiency, as outlined in, e.g., Mallén-Ornelas et al. (2003), Pepper \& Gaudi (2005), and von Braun et al. (2005). Furthermore, individual astrophysical parameters can have an influence on the window function and associated detection probability. In this Section, we show the effects of a number of different parameters, both in the presense (black line) and absence (grey line) of red noise.

\subsection{Observing Run Length}

Transit surveys that are not able to use their own dedicated equipment will often be limited by the number of observing nights allocated to their programs. Understanding whether or not one will reach a certain probability of transit detectability in one's data after a given number of nights is important. Furthermore, one may consider doubling the number of monitored stars by dividing a long observing run into two parts and changing targets after a certain number of nights. An estimate of how much the change in number 

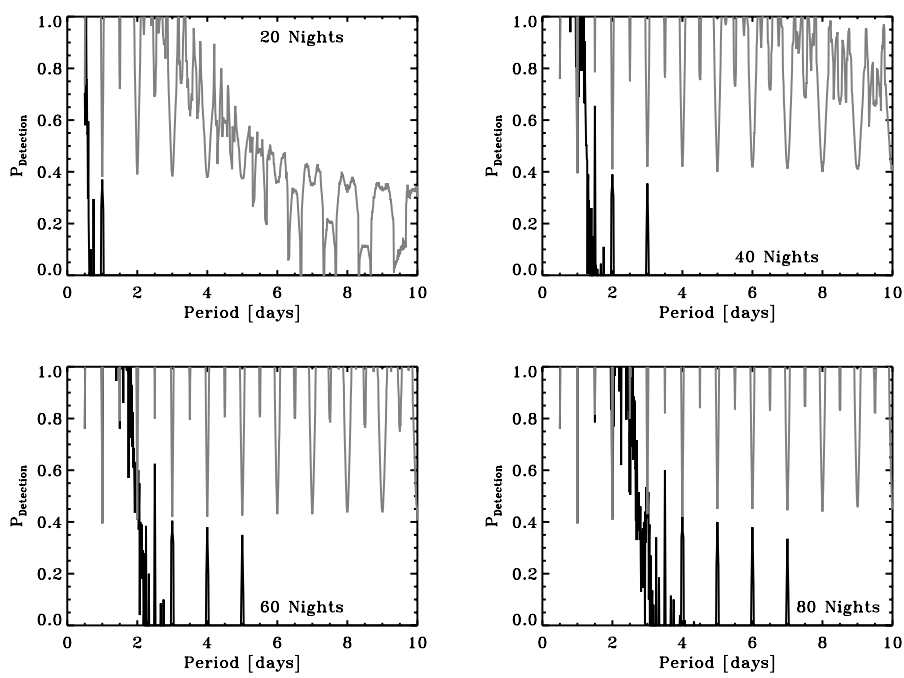

Figure 3. Length of observing run measured in number of 8-hour-long consecutive nights (indicated in each panel), both in the absence (grey line) and presence (black line) of red noise $\left(\sigma_{r}=3 \mathrm{mmag}\right)$. Other input parameters are a 10-min observing cadence, 8-hour-long nights, $\sigma_{w}=5 \mathrm{mmag}, \mathrm{SNR}_{\text {threshold }}=10$, and stellar/planetary radius $=1.0 / 0.1 \mathrm{R}_{\text {sun }}$. Changing the observing run length has a signficant effect on the transit detection efficiency.

of observing nights influences the projected survey yield is crucial to the design of a successful transit survey.

Fig. 3 shows the detection probability for a range in observing run lengths, measured in number of consecutive nights of 8 hours in length with an observing cadence of 10 min. This confirms earlier results in the literature, e.g., von Braun et al. (2005) (based on zero red noise assumptions) that target field switching during observing runs can be detrimental for projected survey yields despite the increase in monitored stars.

\subsection{Observing Cadence}

For a given telescope/detector combination, the observing cadence would change as a function of target brightness (to reduce white noise and avoid saturation). One could alternatively, for a given exposure time, conceive of a survey strategy where one would move back and forth between pointings to increase the number of monitored stars (as an alternative to $\S 4.1$ for, e.g., two separate fields that are near each other on the sky).

Fig. 4 shows that the change in cadence greatly affects white noise dominated regimes (grey line for $\sigma_{r}=0$ ), but shows a relatively weak influence when red noise is present. It should be pointed out, however, that frequently changing between targets during the night will most likely introduce a red noise component by itself.

\subsection{Length of Night}

The number of hours that a target field can be observed during the course of a night depends on the combination of its coordinates, the location of the observatory, and the time of year. Weather can also be a (more random) factor. Finally, one may choose, again, to increase the number of monitored stars by splitting the observing up into multiple target fields during the night (as an alternative to the strategies described in $\S 4.1$ and $\S 4.2$ ). The effect of changing the length of night upon detection efficiency is illustrated in Fig. 5, showing that, e.g., splitting the night in half to double the number 

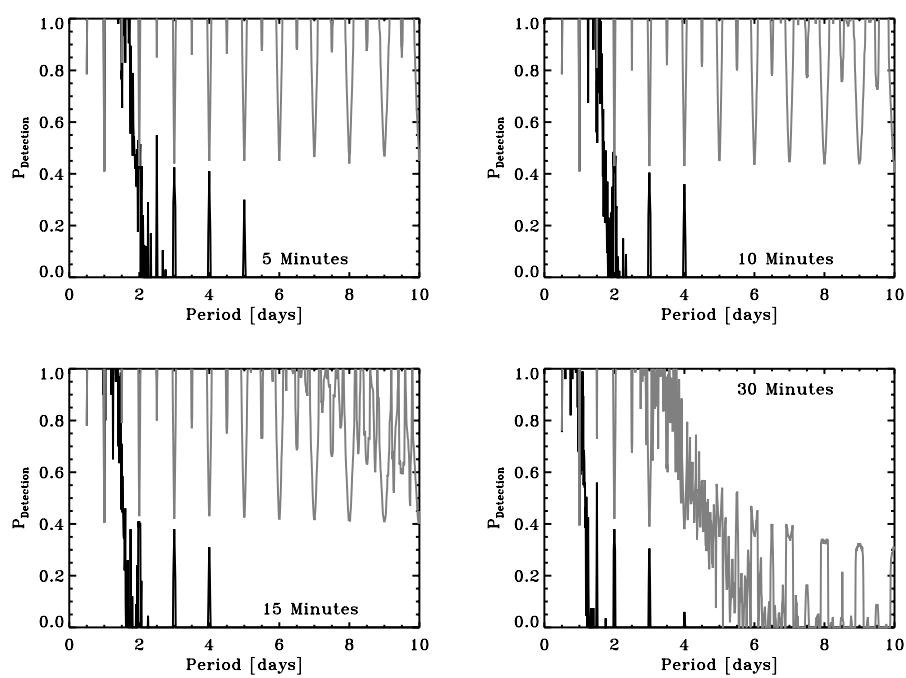

Figure 4. Observing cadence with (black line) and without (grey line) red noise ( $\sigma_{r}=3 \mathrm{mmag}$ ). The effect of changing observing cadence is more pronounced in the white dominated regime. Other input parameters are 50 consecutive 8-hour-long nights of observing, $\sigma_{w}=5 \mathrm{mmag}$, $\mathrm{SNR}_{\text {threshold }}=10$, and stellar/planetary radius $=1.0 / 0.1 \mathrm{R}_{\text {sun }}$.
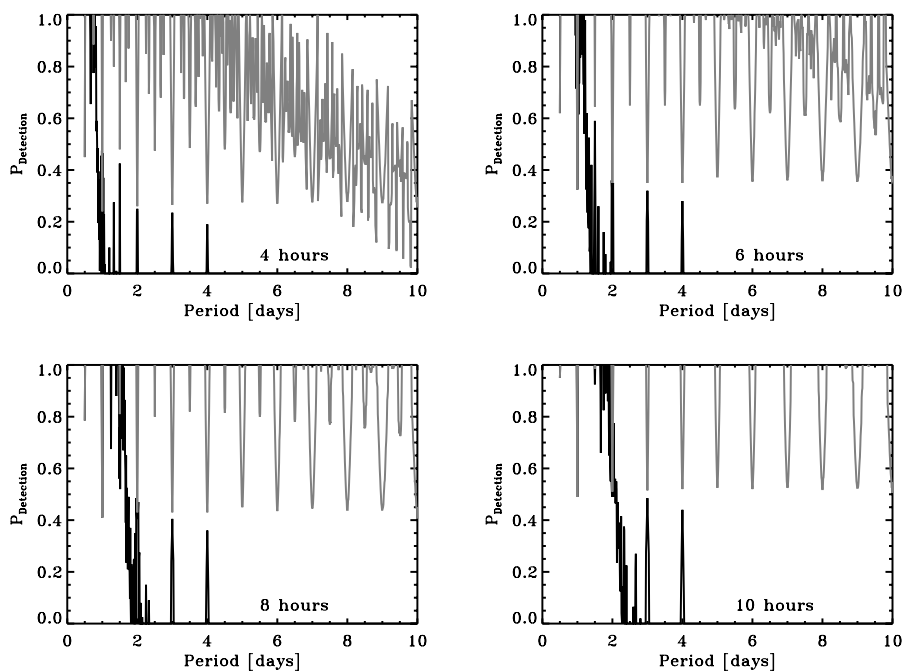

Figure 5. Length of night in continuous hours of observing (indicated in all four panels) in the absence (grey line) and presence (black line) of red noise $\left(\sigma_{r}=3 \mathrm{mmag}\right)$. Other input parameters are 50 consecutive nights of observing, a 10-min observing cadence, $\sigma_{w}=5 \mathrm{mmag}, \mathrm{SNR}_{\text {threshold }}$ $=10$, and stellar/planetary radius $=1.0 / 0.1 \mathrm{R}_{\text {sun }}$. Changing the number of observing hours per night greatly influences transit detection probability.

of monitored stars noticeably decreases the detection probability, especially for orbital periods longer than 2 days, again in agreement the (white noise only) findings in von Braun et al. (2005). 

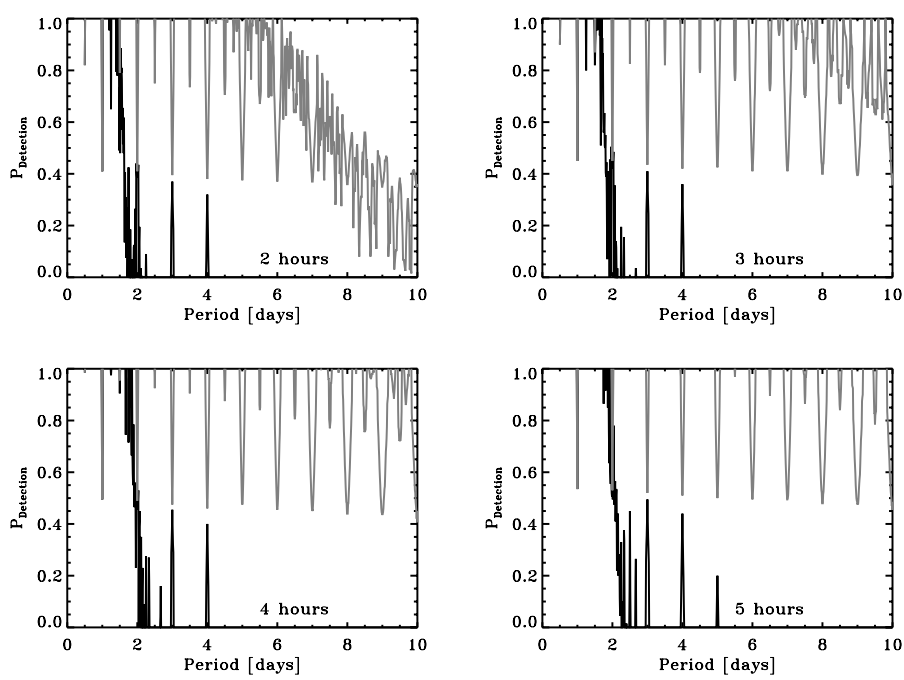

Figure 6. Transit duration in hours (shown in each of the four panels), in the absence (grey line) and presence (black line) of red noise $\left(\sigma_{r}=3 \mathrm{mmag}\right)$. Other input parameters are 50 consecutive nights of observing, a 10-min observing cadence, $\sigma_{w}=5 \mathrm{mmag}$, $\mathrm{SNR}_{\text {threshold }}=10$, and a transit depth of 0.01 mag. The extent to which the detection probability is affected by transit duration is higher for white noise dominated data.

\subsection{Transit Duration}

The duration of a transit is dependent upon the combination of stellar and planetary radii, the orbital period, and the inclination angle of the system, as described in, e.g., Mandel \& Agol (2002) and Seager \& Mallén-Ornelas (2003). To simulate the effect of a non-central transit, Fig. 6 shows how the detection probability changes as the duration of transit varies between 2 and 5 hours, typical for planets with periods of around a few days. The impact of shorter transit durations is noticeably higher in the white noise dominated regime (grey line). This appears to indicate that, as long as the system actually transits, the dependence of the detection efficiency upon inclination angle is relatively weak.

\section{Summary and Conclusions}

In this presentation, we illustrate the influence of several parameters on the probability that an existing planetary transit is detectable in a data set with given noise properties. Red noise dominates in the regime in which planets are typically found (the brightest stars in one's sample). In order to beat down red noise effects and improve detection efficiency, detrending is a vital instrument.

Transit survey strategy can be employed to maximize the projected yield of a given survey. We examine how much the detection efficiency for different orbital periods would suffer when changing one's observing strategy, e.g., to increase the number of monitored stars. We show examples involving the number of consecutive observing nights, typical night length, as well as the observing cadence, and we find that sacrificing full nights or parts of nights can significantly lower transit detection probability. Finally, we show the effects of non-central transits and the associated change of transit duration upon detection efficiency. Our results indicate that, for $\sigma_{r}=3 \mathrm{mmag}$ (typically found in some of the major transit surveys before detrending is applied), small changes in inclination angle and associated transit duration do not greatly affect detection efficiency. 
In all simulations presented here, the presence of red noise (for $\sigma_{r}=3 \mathrm{mmag}$ ) dominates all other effects. However, the variation of some of the parameters examined in this work (in particular the observing cadence and the transit duration) appears to have a bigger effect on white noise dominated (e.g., detrended) data.

\section{References}

Aigrain, S. \& Pont, F. 2007, MNRAS, 378, 741

Kane, S. R., et al. 2007, ArXiv e-prints, 711, arXiv:0711.2581

Kovács, G., Bakos, G., \& Noyes, R. W. 2005, MNRAS, 356, 557

Mandel, K., \& Agol, E. 2002, ApJL, 580, L171

Mallén-Ornelas, G., Seager, S., Yee, H. K. C., Minniti, D., Gladders, M. D., Mallén-Fullerton, G. M., \& Brown, T. M. 2003, ApJ, 582, 1123

Pepper, J., \& Gaudi, B. S. 2005, ApJ, 631, 581

Pont, F. 2006, in "Tenth Anniversary of 51 Peg-b: Status of and prospects for hot Jupiter studies" (eds. Arnold, L., Bouchy, F., \& Moutou, C.), p.153 (astro-ph/0612540)

Pont, F., Zucker, S., \& Queloz, D. 2006, MNRAS, 373, 231

Seager, S., \& Mallén-Ornelas, G. 2003, ApJ, 585, 1038

Tamuz, O., Mazeh, T., \& Zucker, S. 2005, MNRAS, 356, 1466

von Braun, K., Lee, B. L., Seager, S., Yee, H. K. C., Mallén-Ornelas, G., \& Gladders, M. D. 2005, PASP, 117, 141 